\title{
Images of the Rat bone, Vertebra and Test phantom Using Diffraction-Enhanced Imaging Technique with 20,30 and 40 keV Synchrotron X-rays
}

\author{
Donepudi V. Rao ${ }^{1}$, Zhong Zhong ${ }^{2}$, Tetsuya Yuasa ${ }^{3}$, Takao Akatsuka ${ }^{3}$, \\ and Tohoru Takeda ${ }^{4}$, Giuliana Tromba ${ }^{5}$ \\ ${ }^{1}$ Department of Physics, Sir. C.R.R. (A) College, Eluru-534007., W.G. Dt., A.P., India. \\ ${ }^{2}$ National Synchrotron Light Source, NSLS, Brookhaven National Laboratory, Upton, NY 11973, USA. \\ ${ }^{3}$ Dept of Bio-System Engineering, Faculty of Engineering, Yamagata University, 4-3-16 Jonan, Yonezawa 992-8510, \\ Japan \\ ${ }^{4}$ Institute of Clinical Medicine, University of Tsukuba, Tsukuba, Japan \\ ${ }^{5}$ Synchrotron Radiation for Medical Physics, Elettra, Trieste, Italy
}

\begin{abstract}
Images of rat bone of different age groups (8, 56 and 78 weeks), lumbar vertebra and calcium hydroxyapatite phantom are obtained utilizing the diffraction-enhanced imaging technique. Images obtained with DEI are of superior quality and this novel technique may be an excellent choice for better visualization of the microstructure and the embedded spongiosa. Our motivation is to develop the optimizing tomography with the use of the data obtained at multiple energies.
\end{abstract}

Keywords: Images, rat bone, vertebra, DEI, synchrotron $x$-rays.

PACS: 87, 87.57.-e, 87.59.Nk

\section{MOTIVATION}

$\mathrm{X}$-ray imaging techniques were applied extensively to study the 3D microstructure of the bone and embedded internal features. Experimental studies are confined to the conventional techniques with the use of the tube source of $\mathrm{X}$-rays. Conventional X-ray imaging technique utilizing the tube source of X-rays relies on the absorption variation in the object to give a gray scale picture. The flux emitted by the X-ray tube is considerably low and associated bremsstrahlung radiation reduces the required monochromicity. An ideal monoenergetic radiation for this purpose comes from a synchrotron source since it provides considerable monochromicity with high flux. Recently, refraction properties of X-rays turn to be more attractive advantages for imaging over the absorption properties. Refraction is orders of magnitude more sensitive, particularly for biological or low materials (Zhong 2000). The Diffractionenhanced imaging (DEI) technique exploits these refraction for differentiating the embedded spongiosa with high collimated synchrotron X-rays [1].

With the introduction of diffraction-enhanced X-ray imaging (DEI) which is capable of rendering images with absorption, refraction and scatter rejection qualities has allowed detection of specific soft tissues based on small differences in tissue densities. This novel X-ray imaging technology carried out at NSLS, BNL has previously shown it is possible to visualize breast tissue and a number of related medical samples demonstrate that the technique has the potential to provide new information [2-9].

For the last few years, DEI is continuously and consistently upgraded with the inclusion of new optics, electronics, mechanics and mountings and software at various stages at NSLS, BNL, USA, before imaging. Improvements in image quality were carried out regularly on phantoms and biological samples. These studies suggest that, DEI is a potential, valuable and reliable tool for studying the refraction and scattering properties. Diffraction-enhanced imaging technique derives contrast from absorption, refraction and extinction. Real and imaginary parts of the refractive indices are responsible for the phase-shifts and absorption for enhanced contrast imaging. For biological samples, the real part is up to 1000 times greater than the imaginary part. In this context, 
phase-sensitive imaging techniques are more efficient than conventional methods. Images are acquired using 20, 30 and $40 \mathrm{keV}$ synchrotron $\mathrm{X}$-rays.

In view of this, we applied this novel technique for the rat bone of different age groups and lumbar vertebra with spongiosa. The main motivation and the real thrust of this study is to utilize the DEI to image the above samples in order to produce both pure absorption and refraction images. Monochromaticity of these synchrotron X-rays and the associated DEI system, improved the contrast of the images considerably. The choice of optimum energy is chosen based on the quality of the image for better visualization and analysis.

\section{Materials and Methods}

The details of DEI technology and the associated instrumentation have been presented previously and here we provide a brief description.

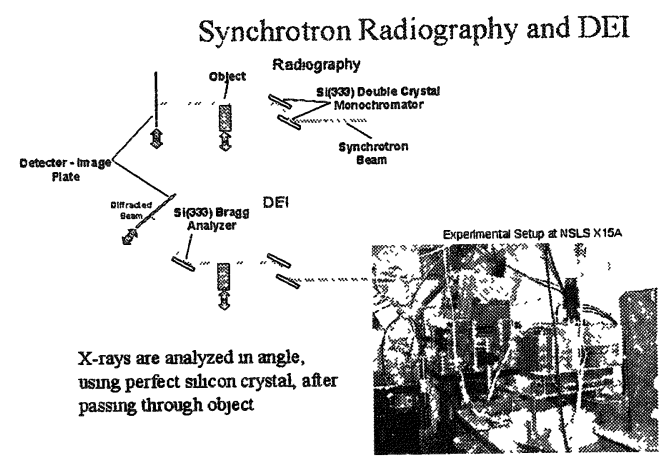

FIGURE 1. Experimental arrangement and the associated DEI system

The experimental arrangement is shown in Fig. 1. The collimated fan beam of X-rays is prepared by the Silicon $[3,3,3]$ monochromator consisting of two perfect silicon crystals. Once this beam passes through the subject, a third crystal (analyzer crystal) of the same reflection index diffracts the X-rays onto radiographic film (Kodak Professional Industrie 150, Industrex SR45) or an image plate detector (Fuji HRV image plate, read out by a Fuji BAS2500 image plate reader). The distance between the X-ray source and the specimen is approximately $20 \mathrm{~m}$ while the distance between the specimens and the X-ray film or image plate detector is $1 \mathrm{~m}$. The image of the subject is formed by scanning the subject and X-ray film at the same speed through the fan beam, in approximately opposite directions to take account of the Bragg reflection by the analyzer crystal. Because of the non-dispersive nature of the crystals, the narrow Darwin-width of the diffraction used, and the small distance between the sample and the detector, the resolution of the image obtained is limited by the resolution of the X-ray film, which is approximately $50 \mu \mathrm{m}$, or the pixel size of the image plate detector, which is approximately $75 \mu \mathrm{m}$. The Bragg condition for the analyzer crystal is met only when the incident beam makes the correct angle with the lattice planes in the crystal for a given X-ray energy. When this condition is met, the beam diffracts from the planes over a narrow range of incident angles. As the analyzer crystal is rotated about a horizontal plane, the crystal will go through a Bragg condition for diffraction and the diffracted intensity will trace out a profile or a rocking curve. The rocking curve of the analyzer in the protocol described here is roughly triangular and has peak intensity close to that of the beam striking in it. The width of this profile is typically a few micro radians (the full width at half maximum is 1.5 micro radians at an X-ray energy of $40 \mathrm{keV}$ and 3.6 micro radians at $18 \mathrm{keV}$, using the $\mathrm{Si}[3,3,3]$ reflection). This narrow angular width provides the tools necessary to prepare and analyze, on the micro radian scale, the angle of $\mathrm{X}$ ray beams modified by the subject while traversing it. Since the range of the angles that can be accepted by the analyzer crystal is only a few micro radians, the analyzer crystal detects the subject's X-ray scattering (ultra-smallangle-scattering) and refraction of X-rays at the micro radian level, an angular sensitivity which is not possible in conventional radiography. The X-ray intensity in the subject is therefore modulated by the scattering and refraction properties of the subject. To extract refraction information, the analyzer is typically set to the half intensity points on the low-and high angle sides of the rocking curve refereed to as -1 and +1 , respectively, in the following discussion), or at the base of the rocking curve (refereed as -2 and +2 , respectively, for the low- and high angle sides), while the imaging takes place. For optimal extinction (scatter rejection) sensitivity, the analyzer is typically set to the peak of the rocking curve during imaging. The reproducibility of the $\mathrm{DEI}$ images is maintained by monitoring the intensity 
of the diffracted X-rays by the analyzer just prior to imaging, to ensure that the analyzer is at the prescribed angular position.

\section{Specimens}

Bone phantom's with different calcium hydroxyapatite concentrations $(10 \mathrm{~mm} \times 10 \mathrm{~mm}, 10 \mathrm{~mm} \times 8 \mathrm{~mm})$, rat's vertebra and bone samples of different age groups $(8,56$ and 78 weeks) were prepared and stored. The diameter of the cylindrical phantoms varies from 8 to $10 \mathrm{~mm}$.

\section{RESULTS AND DISCUSSION}

Figures 2 to 4 shows the images of the rat bone of different age groups, calcium hydroxyapatite phantom and the rat's vertebra at 20,30 and $40 \mathrm{keV}$. In order to visualize the interior microstructure and for sharp images we acquired the images at different energies and the choice of optimum energy is chosen based on the quality of the image. The contrast in the refraction image represents differences in intensity scattered through a particular angle. Sources of contrast in the apparent absorption image are absorption and extinction in scatter image. Extinction here means missing intensities of those X-rays that are refractive under angles substantially higher than the acceptance of the analyzer. The interior microstructure including the embedded spongiosa is sharper with high contrast. As regards to the rat's lumber vertebra, we differentiated the central portion of the vertebra at different orientations for clear visualization of the external and internal features. Expect in few orientations, majority of the images are acceptably distinct at $40 \mathrm{keV}$ and the system is more sensitive and reliable at this optimum energy. The visibility of porosity is considerably large at $40 \mathrm{keV}$ has mesh compared to 20 and $30 \mathrm{keV}$. The porosity of the phantom (differences in density) at $40 \mathrm{keV}$ has closed mesh structure compared to 20 and $30 \mathrm{keV}$.

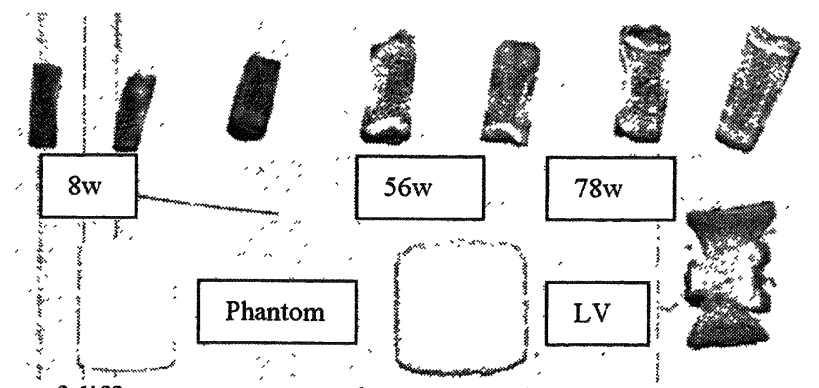

FIGURE 2. Images of the rat bone of different age groups, phantom and the vertebra (LV) with $20 \mathrm{keV}$ synchrotron X-rays.

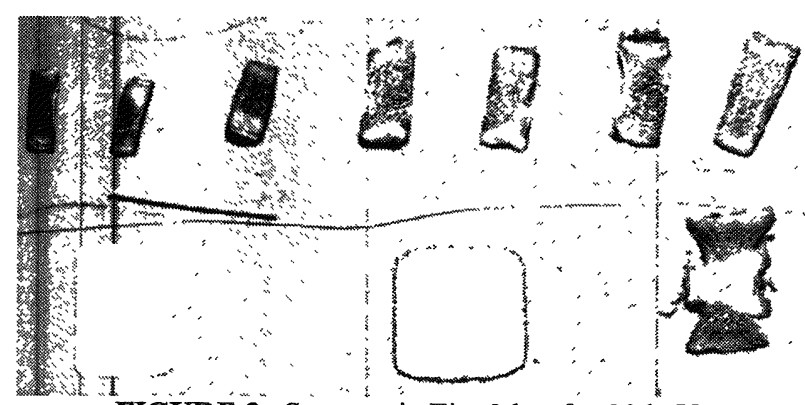

FIGURE 3. Same as in Fig. 2 but for $30 \mathrm{keV}$. 


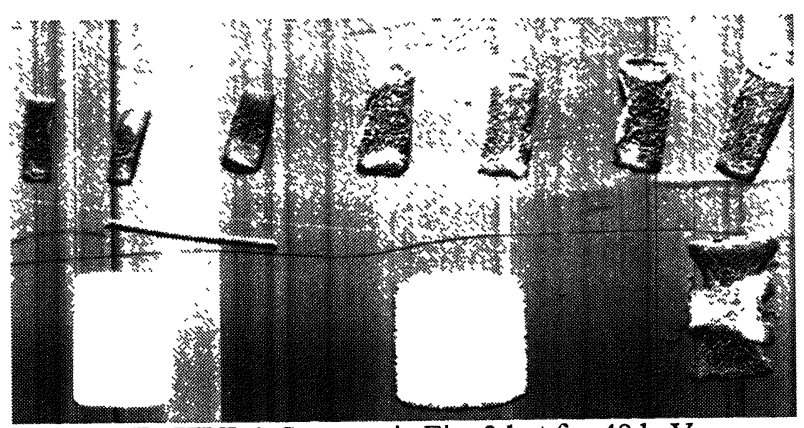

FIGURE 4. Same as in Fig. 2 but for $40 \mathrm{keV}$

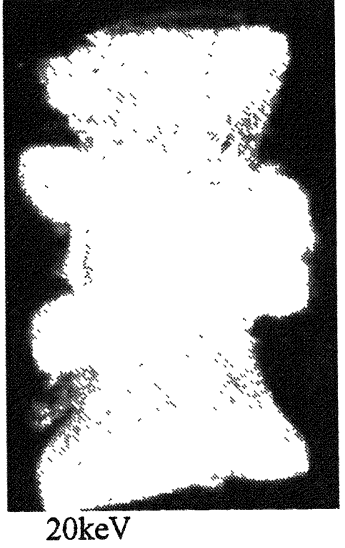

(a)

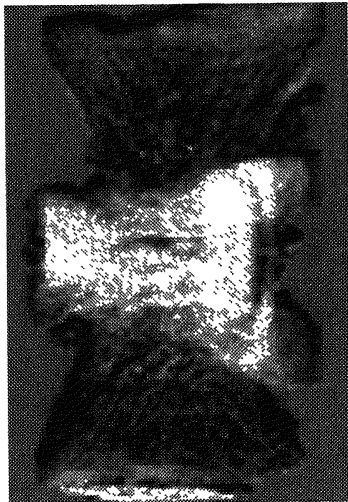

$30 \mathrm{keV}$

(b)

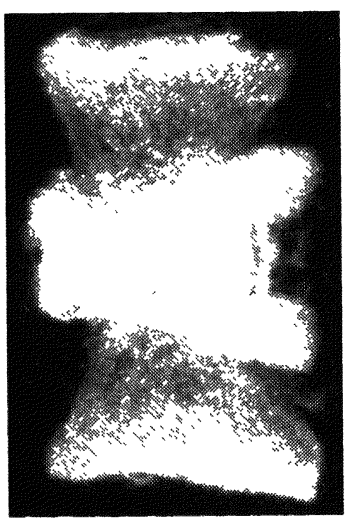

$40 \mathrm{keV}$

(c)

FIGURE 5. Images of the vertebra for 20,30 and $40 \mathrm{keV}$ synchrotron $\mathrm{X}$-rays

Figure 5 shows the images of vertebra obtained at $40 \mathrm{keV}$. The interior microstructure including the embedded spongiosa is sharper with high contrast. As regards to the rat's vertebra, we differentiated the central portion of the vertebra at different orientations for clear visualization of the external and internal features. Except in few orientations, the majority of the images are acceptably distinct at $40 \mathrm{keV}$ and the system is more sensitive and reliable at this optimum energy. The image of the lumber vertebra is clearly visible with its associated microstructure in the middle, internal features and also the porosity.

\section{ACKNOWLEDGEMENTS}

One of the author's (DVR) undertook part of this work with a support from Department of Bio-System Engineering, Yamagata University, Yonezawa, and JSPS, Japan. In addition the potential author received per-diem from BNL and collaboration with Z. Zhong.

\section{REFERENCES}

1. Z. Zhong, W. Thomlinson, D. Chapman, and D. Sayers, NIM A 450, 556-567, 2000.

2. R .E. Johnston, D. Walburn, P. Pisano, C. Bums, W. C. Thomlinson, D.L. Chapman, F. Arfelli, N. Gmür, N., Z. Zhong, D. Sayers, Radiology 200, 659-663,1996.

3. D. Chapman, W. Thomlinson, R.E. Johnston, D. Washburn, E. Pisano, N. Gmür, Z. Zhong, R., Menk, F. Arfelli, D. Sayers, Phys. Med. Biol. 42, 2015-2025,1997.

4. D. Chapman, W. Thomlinson, Z. Zhong, R. E. Johnston, E. Pisano, D. Washburn, D. Sayers, C. Segre, Synchrotron Radiat. News 11 (2) 4, 1998.

5. D. Chapman, E. Pisano, W. Thomlinson, Z. Zhong, R. E. Johnston, D. Washburn, D. Sayers, Breast Dis. 10, 197-207, 1998a.

6. C. Muehleman, D. L. Chapman, K. E. Kuettner, J. Rieff, J. A. Mollenhauer, K. Massuda, and Z. Zhong, The Anatomical Record part A 272, 392-397, 2003.

7. O Oltulu, Z. Zhong, M. Hasnah, M. N. Wernick, and D. Chapman, J. Phys. D: Appl. Phys. 36, 2152-2156, 2003.

8. J. Li, Z. Zhong, R. Lidtke, K.E.Kuettner, C. Peterfy, E. Aliyeva, and C. Muehleman, J. Ant. 202, 463-470, 2003.

9. M. Z. Kiss, D. E. Sayers, Z. Zhong, C. Parham, and E. Pisani, Phys. Med. Biol. 49, 3427-3439, 2004. 